\title{
NONEXISTENCE OF $2-(v, k, 1)$ DESIGNS ADMITTING AUTOMORPHISM GROUPS WITH SOCLE $E_{8}(q)$
}

\begin{abstract}
SHANGZHAO LI
Received 21 December, 2014

Abstract. One of the outstanding problems in combinatorial design theory is concerning the existence of $2-(v, k, 1)$ designs. In particular, the existence of $2-(v, k, 1)$ designs admitting an interesting group of automorphisms is of great interest. Thirty years ago, a six-person team classified $2-(v, k, 1)$ designs which have flag-transitive automorphism groups. Since then the effort has been to classify those $2-(v, k, 1)$ designs which are block-transitive but not flagtransitive. This paper is a contribution to this program and we prove there is nonexistence of $2-$ $(v, k, 1)$ designs admitting a point-primitive block-transitive but not flag-transitive automorphism group $G$ with socle $E_{8}(q)$.
\end{abstract}

2010 Mathematics Subject Classification: Primary 05B05; Secondary 20B25

Keywords: block-transitive, point-primitive, design, socle

\section{INTRODUCTION}

This paper is part of a project to classify groups and $2-(v, k, 1)$ designs where the group acts transitively on the blocks of the design. A $2-(v, k, 1)$ design $\mathscr{D}=(\mathcal{P}, \mathscr{B})$ is a pair consisting of a finite set $\mathcal{P}$ of points and a collection $\mathscr{B}$ of $k$-subsets of $\mathcal{P}$, called blocks, such that any 2 -subsets of $\mathcal{P}$ is contained in exactly one block. Traditionally one defined $v=:|\mathcal{P}|$ and $b=:|\mathscr{B}|$. We will always assume that $2<$ $k<v$.

One of the outstanding problems in combinatorial design theory is concerning the existence of $2-(v, k, 1)$ designs. In particular, the existence of $2-(v, k, 1)$ designs admitting an interesting group of automorphisms is of great interest. Thirty years ago, a six-person team [2] classified the pairs $(\mathscr{D}, G)$ where $\mathscr{D}$ is a $2-(v, k, 1)$ design and $G$ is a flag-transitive automorphism group of $\mathscr{D}$, with the exception of those in which $G$ is a one-dimensional affine group. Since then the effort has been to classify those $2-(v, k, 1)$ designs which are block-transitive but not flag-transitive. These fall naturally into two classes, those where the action on points is primitive and those where the action on points is imprimitive. The primitive ones are now subdivided,

The author was supported in part by the National Natural Science Foundation of China, Grant Nos. 11471054 and 11271208. 
according to the O'Nan-Scotte theorem and some further work by Camina, into the socles which are either elementary abelian or non-abelian simple. As a result of [6] it is known that the second only occur finitely times for a given line size. This paper contributes to the program for determining the pairs $(\mathscr{D}, G)$ in which $\mathscr{D}$ has a pointprimitive block-transitive subgroup, $G$, of automorphisms. From the assumption that $G$ is transitive on the set $\mathscr{B}$ of blocks, it follows that $G$ is also transitive on the point set $\mathcal{P}$. This is a consequence of the theorem of Block in [1].

The classification of block-transitive $2-(v, 3,1)$ designs was completed about thirty years ago (see [4]). In [3] Camina and Siemons classified 2- $(v, 4,1)$ designs with a block-transitive, solvable group of automorphisms. Li classified $2-(v, 4,1)$ designs admitting a block-transitive, unsolvable group of automorphisms (see [11]). Tong and Li classified $2-(v, 5,1)$ designs with a block-transitive, solvable group of automorphisms in [19]. Liu classified $2-(v, k, 1)$ (where $k=6,7,8,9,10)$ designs with a block-transitive, solvable group of automorphisms in [16]. Ding [8] considered $2-(v, k, 1)$ designs admitting block-transitive automorphism groups in $A G L(1, q)$ and prove the existence of $2-(v, 6,1)$ designs which have block-transitive but not flag-transitive automorphism groups in $A G L(1, q)$ (see [7]). Dai and Zhao consider $2-(v, 13,1)$ designs with point-primitive block-transitive unsolvable group of automorphisms whose socle is $S z\left(2^{2 n+1}\right)$ in [5]. Recently, there have been a number contributions to this classification (see $[13,14]$ ). Here we focus on the existence problem of $2-(v, k, 1)(k \leq 2793)$ designs with a point-primitive block-transitive automorphism group of almost simple type and prove the following theorem:

Theorem 1. Suppose that $E_{8}(q) \unlhd G \leq A u t\left(E_{8}(q)\right)$ for $q>5$. Then there is nonexistence of $2-(v, k, 1)(k \leq 2793)$ design $D$ admitting a point-primitive blocktransitive but not flag-transitive automorphism group $G$.

We introduce some notation below. Let $X$ and $Y$ be arbitrary finite groups. The expression $X \cdot Y$ denotes an extension of $X$ by $Y$ and $X: Y$ denotes the split extension. If $Y$ is a subgroup of $X$, then the symbol $|X: Y|$ denotes the index of $Y$ in $X$. Let $\mathscr{D}$ be a $2-(v, k, 1)$ design and $G$ be an automorphism group of $\mathscr{D}$. If $B$ is a block, then $G_{B}$ denotes the setwise stabilizer of $B$ in $G$ and $G_{(B)}$ is the pointwise stabilizer of $B$ in $G$. In addition, $G^{B}$ denotes the permutation group induced by the action of $G_{B}$ on the points of $B$. Then $G^{B} \cong G_{B} / G_{(B)}$. We will write $\alpha$ to be a point of $\mathscr{D}$ and $G_{\alpha}$ to be the stabilizer of $\alpha$ under the action of $G$. Other notation for group structure is standard.

The paper is organized as follows. Section 2 describes several preliminary results concerning the group $E_{8}(q)$ and $2-(v, k, 1)$ designs. Section 3 gives the proof of the theorem. 


\section{Preliminary RESUlts}

Suppose that $G$ is a block-transitive automorphism group of a $2-(v, k, 1)$ design. It is well-known that:

$$
\begin{gathered}
v=r(k-1)+1 ; \\
v(v-1)=b k(k-1) .
\end{gathered}
$$

Then we have $r=(v-1) /(k-1)$. We can show that $b \geq v$ and so $k \leq r$. If $k=r$ then $v=k^{2}-k+1$; if $r \geq k+1$, then $v \geq k^{2}$.

We use a result of W. Fang and H. Li [9]. Define the following constants:

$$
b_{1}=(b, v), b_{2}=(b, v-1), k_{1}=(k, v) \text {, and } k_{2}=(k, v-1) .
$$

Using the basic equalities 2.1 and 2.2, we get the Fang-Li Equations:

$$
k=k_{1} k_{2}, b=b_{1} b_{2}, r=b_{2} k_{2} \text {, and } v=b_{1} k_{1} .
$$

We shall state a number of basic results which will be used repeatedly throughout the paper. Liebeck and Saxl have determined the maximal subgroups of $\operatorname{Soc}(G)=$ $E_{8}(q)$ in [15].

Lemma 1 ([15]). Suppose that $T=E_{8}(q) \unlhd G \leq A u t(T)$. Let $M$ be a maximal subgroup of $G$ not containing $T$. Then one of the following holds

(1) $|M|<q^{110}|G: T|$;

(2) $M \cap T$ is a parabolic group;

(3) $M \cap T$ is isomorphic to $\left(S L_{2}(q) \circ E_{7}(q)\right) . d, D_{8}(q) . d$, or $E_{8}\left(q^{\frac{1}{2}}\right)$ with $q$ square, where $d=(2, q-1)$.

Lemma 2 ([18]). Let $G=T:\langle x\rangle$ and act block-transitively on a $2-(v, k, 1)$ design $\mathscr{D}=(\mathcal{P}, \mathcal{B})$, where $x \in \operatorname{Out}(T)$. Then $T$ acts transitively on $\mathcal{P}$.

Lemma 3 ([17]). Let $G$ be a solvable block-transitive automorphism group of a $2-(v, k, 1)$ design. If $G$ is point-primitive, then

(1) there exists a prime number $p$ and a positive integer $n$ such that $v=p^{n}$;

(2) if there exists a p-primitive prime divisor e of $p^{n}-1$, such that e ||$G \mid$, then either $G \leq A \Gamma L\left(1, p^{n}\right)$ or $k \mid v$.

Lemma 4 ([10]). Let $\mathfrak{D}$ be a $2-(v, k, 1)$ design admitting a block-transitive and point-primitive but not flag-transitive automorphism group $G$. Assume that $T=$ $\operatorname{Soc}(G)$ and $T_{\alpha}=T \cap G_{\alpha}$ where $\alpha \in \mathcal{P}$. Then the following hold:

(1) $\frac{v}{z}<\left(k_{2} k-k_{2}+1\right)|G: T|$, where $z$ is the size of a $T_{\alpha}-$ orbit in $\mathcal{P} \backslash\{\alpha\}$;

(2) if $(v-1, q)=1$, then there exists a $T_{\alpha}$-orbit with size $y$ in $\mathcal{P} \backslash\{\alpha\}$ such that $\left.y|| T_{\alpha}\right|_{p^{\prime}}$.

Lemma 5. Let $\mathfrak{D}$ be a $2-(v, k, 1)$ design admitting a block-transitive automorphism group $G$. Assume that $T=\operatorname{Soc}(G)$ and $T_{\alpha}=T \cap G_{\alpha}$ where $\alpha \in \mathcal{P}$. Then

(1) $v=k_{2}(k-1) b_{2}+1$; 
(2) $\left.b_{2}|| T_{\alpha}\right|_{v^{\prime}}|G: T|$ and $v \leq 1+k(k-1)\left|T_{\alpha}\right|_{v^{\prime}}|G: T|$;

(3) If $G$ is not flag-transitive and non-solvable, then $\frac{|T|}{\left|T_{\alpha}\right|^{2}} \leq \frac{k(k-1)+1}{2}|G: T|$.

Proof. (1) Since $k(k-1) b=v(v-1)$ and $k=k_{1} k_{2}, b=b_{1} b_{2}, v=b_{1} k_{1}$, we obtain $k_{2}(k-1) b_{2}=v-1$ and hence $v=1+k_{2}(k-1) b_{2}$.

(2) Since $r v=b k$, it follows that $r\left|G: G_{\alpha}\right|=k\left|G: G_{B}\right|$, where $\alpha \in \mathcal{P}, B \in \mathscr{B}$. Recall that $k=k_{1} k_{2}, r=b_{2} k_{2}$. It is clear that $b_{2}\left|G_{B}\right|=k_{1}\left|G_{\alpha}\right|$. Note that $\left(b_{2}, k_{1}\right)=$ 1 and hence $b_{2}$ divides $\left|G_{\alpha}\right|$. Since $\left(b_{2}, v\right)=1$, then $\left.b_{2}|| G_{\alpha}\right|_{v^{\prime}}$. Since $G$ is blocktransitive, by Lemma 2, $T$ is point-transitive. We conclude that $v=\left|G: G_{\alpha}\right|=\mid T$ : $T_{\alpha} \mid$. Hence $\left|G_{\alpha}\right|=\left|T_{\alpha}\right||G: T|$ and so $\left.b_{2}|| T_{\alpha}\right|_{v^{\prime}}|G: T|$. Together with (1), it deduces that $v \leq 1+k_{2}(k-1)\left|T_{\alpha}\right|_{v^{\prime}}|G: T|$ and hence $v \leq 1+k(k-1)\left|T_{\alpha}\right|_{v^{\prime}}|G: T|$.

(3) Let $B$ be a block of $\mathcal{D}$. Since $G$ is non-solvable, the following possibility for the structure of $G^{B}$, the rank and subdegree of $G$ does not occur:

\begin{tabular}{ccc}
\hline Type of $G^{B}$ & Rank of $G$ & Subdegree of $G$ \\
\hline$\langle 1\rangle$ & $1+k_{2}(k-1)$ & $1, \overbrace{b_{2}, b_{2}, \cdots, b_{2}}^{k_{2}(k-1)}$ \\
\hline
\end{tabular}

Otherwise, $\left|G^{B}\right|$ is odd, whence $|G|$ is odd and so $G$ is solvable, which contradicts the fact that $G$ is non-solvable. Then by the proof of Proposition 3.1 in [10] the conclusion holds.

Lemma 6 ([12]). Suppose that $\mathscr{D}$ is a $2-(v, k, 1)$ design and $G$ is an almost simple group acting on $D$ block-transitively. Let $G_{\alpha}$ be the stabilizer in $G$ of a point $\alpha$ of $\mathscr{D}$ and suppose the socle $T$ of $G$ is a simple group of Lie type. If the intersection of $G_{\alpha}$ and $T$ is a parabolic subgroup of $T$, then $G$ acts on $D$ flag-transitively.

\section{Proof of TheOREM 1}

Suppose that there exists a $2-(v, k, 1)(k \leq 2793)$ design $\mathscr{D}$ satisfying the conditions of the Main Theorem. We will derive contradictions to prove the Main Theorem.

Since $T=E_{8}(q) \unlhd G \leq \operatorname{Aut}\left(E_{8}(q)\right)$, then $G=T:\langle x\rangle$ and $|O u t(T)|=a$, where $x \in \operatorname{Out}(T)$. Let $o(x)=m$. Then we obtain that $m \mid a$ and $|G|=q^{120}\left(q^{30}-1\right)\left(q^{24}-\right.$ 1) $\left(q^{20}-1\right)\left(q^{18}-1\right)\left(q^{14}-1\right)\left(q^{12}-1\right)\left(q^{8}-1\right)\left(q^{2}-1\right) m$. Since $G$ is point-primitive, $G_{\alpha}$ is the maximal subgroup of $G$ for any $\alpha \in \mathcal{P}$. Then $M=G_{\alpha}$ satisfies one of the three cases in Lemma 1. If $G_{\alpha} \cap T$ is a parabolic subgroup of $T$, then by Lemma 6 we see that $G$ is flag-transitive, which is a contradiction. Therefore, the case (2) in Lemma 1 does not occur and it suffices to consider the following two cases.

Case 3.1: $\left|G_{\alpha}\right|<q^{110}|G: T|$.

Since $G$ is block-transitive, by Lemma $2, T$ is point-transitive. Hence $\left|G_{\alpha}\right|=$ $\left|T_{\alpha}\right||G: T|$ and so $\left|T_{\alpha}\right|<q^{110}$. Then $v=\left|T: T_{\alpha}\right|$ is not a prime power and by Lemma 3 we have that $G$ is non-solvable. Note that $m=|G: T|$. It follows by 
Lemma 5 (3) that

$$
|T| \leq \frac{k(k-1)+1}{2}\left|T_{\alpha}\right|^{2}|G: T| \leq \frac{7798057}{2} q^{220} m
$$

This gives,

$$
\begin{aligned}
\frac{|T|}{q^{220}} & =\frac{\left(q^{2}-1\right)\left(q^{8}-1\right)\left(q^{12}-1\right)\left(q^{14}-1\right)\left(q^{18}-1\right)\left(q^{20}-1\right)\left(q^{24}-1\right)\left(q^{30}-1\right)}{q^{100}} \\
& <\frac{7798057}{2} m .
\end{aligned}
$$

Since

$$
\left(q^{2}-1\right)\left(q^{8}-1\right)\left(q^{12}-1\right)\left(q^{14}-1\right)\left(q^{18}-1\right)\left(q^{20}-1\right)\left(q^{24}-1\right)\left(q^{30}-1\right)>\frac{7}{10} q^{128},
$$

it implies that

$$
\frac{7}{10} q^{8}<\frac{7798057}{2} m
$$

Recall that $m \mid a, q=p^{a}, p \geq 2$. We can conclude therefore that

$$
\frac{7}{10} \cdot 2^{8 a} \leq \frac{7}{10} \cdot p^{8 a}=\frac{7}{10} q^{8}<\frac{7798057}{2} a,
$$

which forces $a \leq 2$. We calculate to obtain all possibilities for the values of $p$ and $a$ satisfying the inequality 3.1: (1) $a=1, p \leq 5$, a prime; (2) $a=2, p=2$. This contradicts $q>5$.

Case 3.2: $\quad G_{\alpha} \cap T$ is case (3) in Lemma 1.

Now we consider three cases.

Subcase 3.2.1: $\quad T_{\alpha}=\left(S L_{2}(q) \circ E_{7}(q)\right) . d$ where $d=(2, q-1)$.

We observe that

$$
\left|T_{\alpha}\right|=q^{64}\left(q^{18}-1\right)\left(q^{14}-1\right)\left(q^{12}-1\right)\left(q^{10}-1\right)\left(q^{8}-1\right)\left(q^{6}-1\right)\left(q^{2}-1\right)^{2}
$$

and

Hence

$$
v=\frac{q^{56}\left(q^{30}-1\right)\left(q^{24}-1\right)\left(q^{20}-1\right)}{\left(q^{10}-1\right)\left(q^{6}-1\right)\left(q^{2}-1\right)}
$$

$$
\left|T_{\alpha}\right|_{v^{\prime}} \leq\left(q^{2}-1\right)^{8}\left(q^{12}+q^{6}+1\right)\left(1+q^{2}+q^{4}+q^{6}+q^{8}+q^{10}+q^{12}\right)<\frac{7}{5} q^{40} .
$$

Since

$$
v=\frac{q^{56}\left(q^{30}-1\right)\left(q^{24}-1\right)\left(q^{20}-1\right)}{\left(q^{10}-1\right)\left(q^{6}-1\right)\left(q^{2}-1\right)}>\frac{1}{50} q^{112},
$$

we can appeal to Lemma 5 (2) to observe that

$$
\frac{1}{50} q^{112}<v \leq 1+k(k-1)\left|T_{\alpha}\right|_{v^{\prime}}|G: T|<1+7798056 \cdot \frac{7}{5} \cdot q^{40} a .
$$


This implies the following inequality

$$
\frac{1}{50} \cdot 2^{72 a} \leq \frac{1}{50} \cdot q^{72}<\frac{1}{2^{40 a}}+7798056 \cdot \frac{7}{5} \cdot a<\frac{4}{5} \cdot 2^{24} a,
$$

which is impossible.

Subcase 3.2.2: $\quad T_{\alpha}=D_{8}(q) . d$, where $d=(2, q-1)$.

We calculate that

$$
\left|T_{\alpha}\right|=\frac{d q^{56}\left(q^{8}-1\right) \prod_{i=1}^{7}\left(q^{2 i}-1\right)}{d_{1}}
$$

and

$$
v=\frac{d_{1} q^{64}\left(q^{30}-1\right)\left(q^{24}-1\right)\left(q^{20}-1\right)\left(q^{18}-1\right)}{d\left(q^{10}-1\right)\left(q^{8}-1\right)\left(q^{6}-1\right)\left(q^{4}-1\right)}
$$

where $d_{1}=\left(4, q^{8}-1\right)$. Since $(v-1, q)=1$, by Lemma 4 (2), there exists in $\mathcal{P} \backslash\{\alpha\}$ a $T_{\alpha}$-orbit of size $y$ such that $y \|\left. T_{\alpha}\right|_{p^{\prime}}$. Hence

$$
y \leq\left|T_{\alpha}\right|_{p^{\prime}} \leq 2\left(q^{8}-1\right) \prod_{i=1}^{7}\left(q^{2 i}-1\right) .
$$

Thus

$$
\begin{aligned}
\frac{v}{y} & \geq \frac{d_{1} q^{64}\left(q^{30}-1\right)\left(q^{24}-1\right)\left(q^{20}-1\right)\left(q^{18}-1\right)}{2 d\left(q^{14}-1\right)\left(q^{12}-1\right)\left(q^{10}-1\right)^{2}\left(q^{8}-1\right)^{3}\left(q^{6}-1\right)^{2}\left(q^{4}-1\right)^{2}\left(q^{2}-1\right)} \\
& >\frac{\frac{1}{10} \cdot q^{108}}{4 \cdot \frac{15}{2} \cdot q^{44}}=\frac{1}{300} q^{64} .
\end{aligned}
$$

Note that $k_{2} \leq k$. We now apply Lemma 4 (1) to conclude that

$$
\frac{1}{300} \cdot 2^{64 a} \leq \frac{1}{300} q^{64}<\frac{v}{y}<(k(k-1)+1)|G: T| \leq 7798057 a<\frac{19}{20} \cdot 2^{23} a,
$$

which is a contradiction.

Subcase 3.2.3: $T_{\alpha}=E_{8}\left(q^{\frac{1}{2}}\right)$.

We obtain that

$$
\left|T_{\alpha}\right|=q^{60}\left(q^{15}-1\right)\left(q^{12}-1\right)\left(q^{10}-1\right)\left(q^{9}-1\right)\left(q^{7}-1\right)\left(q^{6}-1\right)\left(q^{4}-1\right)(q-1)
$$

and

$$
v=q^{60}\left(q^{15}+1\right)\left(q^{12}+1\right)\left(q^{10}+1\right)\left(q^{9}+1\right)\left(q^{7}+1\right)\left(q^{6}+1\right)\left(q^{4}+1\right)(q+1) .
$$

Then it deduces that

$$
\begin{aligned}
\left|T_{\alpha}\right|_{v^{\prime}} \leq & (q-1)^{8}\left(q^{2}+q+1\right)^{4}\left(q^{6}+q^{3}+1\right)\left(1+q+q^{2}+q^{3}+q^{4}\right)^{2} \\
& \cdot\left(1+q+q^{2}+q^{3}+q^{4}+q^{5}+q^{6}\right)\left(1-q+q^{3}-q^{4}+q^{5}-q^{7}+q^{8}\right)<48 q^{44} .
\end{aligned}
$$

Since

$$
v=q^{60}\left(q^{15}+1\right)\left(q^{12}+1\right)\left(q^{10}+1\right)\left(q^{9}+1\right)\left(q^{7}+1\right)\left(q^{6}+1\right)\left(q^{4}+1\right)(q+1)>q^{124} \text {, }
$$


by Lemma 5 (2) this implies that

$$
q^{124}<v \leq 1+k(k-1)\left|T_{\alpha}\right|_{v^{\prime}}|G: T|<1+7798056 \cdot 48 \cdot q^{44} \cdot a .
$$

This leads to the following result

$$
2^{80 a} \leq q^{80}<\frac{1}{2^{44 a}}+7798056 \cdot 48 a<\frac{4}{5} \cdot 2^{29} a,
$$

which gives a contradiction.

This completes the proof of Theorem 1.

\section{ACKNOWLEDGEMENT}

The author is grateful to the editor and reviewer for pointing out errors in the original version of this paper and their valuable comments and suggestions.

\section{REFERENCES}

[1] R. E. Block, "On the orbits of collineation groups," Math. Z., vol. 96, no. 1, pp. 33-49, 1967, doi: 10.1007/BF01111448.

[2] F. Buekenhout, A. Delandtsheer, J. Doyen, P. B. Kleidman, M. W. Liebeck, and J. Saxl, "Linear spaces with flag-transitive automorphism groups," Geom. Dedicata, vol. 36, no. 1, pp. 89-94, 1990.

[3] A. R. Camina and J. Siemons, "Block transitive automorphism groups of $2-(v, k, 1)$ block designs," J. Comb. Theory, Ser. A, vol. 51, no. 2, pp. 268-276, 1989, doi: 10.1016/00973165(89)90052-6.

[4] P. C. Clapham, "Steiner systems with block transitive automorphism groups," Discrete Math., vol. 14, no. 2, pp. 121-131, 1976, doi: 10.1016/0012-365X(76)90055-8.

[5] S. J. Dai and K. Zhao, "Block transitive 2- $(v, 13,1)$ designs and Suzuki groups," Ars Comb., vol. 105, no. 5, pp. 369-373, 2012.

[6] A. Delandtsheer and J. Doyen, "Most block-transitive $t$-designs are point-primitive," Geom. Dedicata, vol. 29, no. 3, pp. 307-310, 1989, doi: 10.1007/BF00572446.

[7] S. F. Ding, "The existence and construction of a family of block-transitive $2-(v, 6,1)$ designs," $J$. Comb. Theory, Ser. A, vol. 116, no. 1, pp. 215-222, 2009.

[8] S. F. Ding and W. J. Liu, "Existence of $2-(v, k, 1)$ designs admitting a block-transitive group of affine type," Electron. J. Comb., vol. 19, no. 3, pp. 1-12, 2012

[9] W. D. Fang and H. L. Li, "A generalisation of Camina-Gagens Theorem," Math. Mag., (Wuhan), vol. 13, no. 13, pp. 437-441, 1993 (in Chinese).

[10] G. G. Han, "Block-transitive $2-(v, k, 1)$ designs and the twisted simple groups ${ }^{2} E_{6}(q)$," Appl. Math. Comput., vol. 242, no. 9, pp. 109-115, 2014, doi: 10.1016/j.amc.2014.05.027.

[11] H. L. Li, "On block-transitive 2- $(v, 4,1)$ designs," J. Comb. Theory, Ser. A, vol. 69, no. 1, pp $115-124,1995$.

[12] H. L. Li and Y. Liu, "Automorphism groups of linear spaces and their parabolic subgroups," $J$. Comb. Theory, Ser. A, vol. 116, no. 1, pp. 1-11, 2009, doi: 10.1016/j.jcta.2008.04.001.

[13] S. Z. Li, S. J. Dai, and L. Y. Jiang, "Unsolvable block-transitive automorphism groups of 2 (v, 31,1) designs," Ars Comb., vol. 117, no. 5, pp. 469-476, 2014.

[14] S. Z. Li, G. G. Han, and W. J. Liu, "Block-transitive $2-(v, k, 1)$ designs and the Chevalley groups $F_{4}(q)$," Appl. Math. Comput., vol. 248, no. 12, pp. 380-385, 2014. 
[15] M. W. Liebeck and J. Saxl, "On the orders of maximal subgroups of the finite exceptional groups of Lie type," Proc. Lond. Math. Soc., vol. 55, no. 2, pp. 299-330, 1987, doi: 10.1093/plms/s3$55^{\circ} 2.299$.

[16] W. J. Liu, "Block transitive $2-(v, k, 1)$ designs," Ph. D. Thesis, Zhejiang University, Hangzhou, 1998.

[17] W. J. Liu, H. L. Li, and C. G. Ma, "Soluble block-transitive automorphism groups of $2-(v, 7,1)$ designs," Adv. Math., vol. 30, no. 1, pp. 56-62, 2001 (in Chinese).

[18] W. J. Liu, S. Z. Li, and L. Z. Gong, "Almost simple groups with socle Ree( $q$ ) acting on finte linear spaces," Eur. J. Comb., vol. 27, no. 6, pp. 788-800, 2006, doi: 10.1016/j.ejc.2005.05.008.

[19] W. W. Tong and H. L. Li, "Solvable block transitive automorphism groups of $2-(v, 5,1)$ designs," Discrete Math., vol. 260, no. 1-3, pp. 267-273, 2003, doi: 10.1016/S0012-365X(02)00763-X.

\section{Author's address}

\section{Shangzhao Li}

Changshu Institute of Technology, School of Mathematics and Statistics, 99 Hushan St., Changshu, China

E-mail address: 1szfd2004@126.com 